\title{
CAN ANY STATIONARY ITERATION USING LINEAR INFORMATION BE GLOBALLY CONVERGENT?
}

G.W. WASILKOWSKI

CUCS-143-80 


\title{
Can Any Stationary Iteration Using Linear Information Be Globally Convergent?
}

\author{
G. W. WASILKOWSKI \\ Camegie. Mellon Universil!. Putshurgh. Pennsitiania
}

\begin{abstract}
Aestanct All known globally wnvergent iterations for the solution of a nonlinear operator equalion $\lambda(x)=0$ are either nonstationary or use nonlinear information. It is asked whether these exists a globally convergent stationary tteration which uses linear information It is proved that even if global convergence is defined in a weak sense, there exists no such ileration for as simple a class of problems as the set of all analytic complex functions having only simple zeros. It is conjectured that even for the class of all real polvnomials which have real simpic zeros there does not exist a globally convergent stationary iteration using linear information.
\end{abstract}

KEY WORDS AND PMRASES: nonlinear equations, linear information. stationary iterations. global convergence

CR CATEGOUIFS: 5.15

\section{Introduction}

Suppose we solve a nonlinear operator equation $f(x)=0$ by an iteration which constructs a sequence of approximations $\left\{x_{1}\right\}$. For most convergence theorems it is assumed that $f$ is sufficiently smooth and starting points are "sufficiently close" to a solution $\alpha$. In practice it is very hard in verify the second assunuption One therefire wants lu use Heralluns which are globally convergent

All known globally convergent iterations are either nonstationary or use nonlinear information. For instance. Laguerre's iteration (see. e.g. Ralston and Rabinowitz |ll) is globally convergent for the class of all real polynumials having unly real ceros. However this iteration uses the degree of the polynomial whose zero is approximaled. This means that Laguerre's iteration uses nonlinear information (see Section 6). An example of a nonstationary tteration using linear information which is globally convergent for analytic operator equations may be adopted from Traub and Wozniakowski [4]. This will be reported in Wasilkowski [6].

However. most commonly used iterations are stationary and use linear information. Therefore it is important to know whether there exist globally collvergent statiunary iterations which use linear information. In this paper we prove that for as simple a class of problems as the set of all analytic complex functions with simple zeros, there exists no such iteration. We conjecture that the same negative result holds even for the class of all real polynomials having real simple zeros.

We summanze the contents of the paper. In Section 2 we remind the reader of the definitions of information and stationary teration withoul memory. In Section 3 we discuss

Permission to copy without fee all or part of this matenal is granted provided that the copies are not made or distributed for direct commercal advantage. the ACM copynght notice and the title of the publication and its date appear. and notice is given that copying is by permission of the Asuxidliun for (imipuling Mathinery. I." copy uherwise, or to republish. reyutres a fee and "ur specific permission

This research was supponed in part by the National Science Foundation under Grant MCS 75-222-55 and the Office of Naval Research under Coniract N00014.76-C-0370. NR 044-422.

This work was done while the authur was on leave from the Universit) of Waraw

Author's present address: Depanment of Mathematics. University of Warsaw. P K I.N. $8 p$ 850. 00 -901 Warsaw Poland

01980 ACM 0004-5411/80/0400-0263 SR0 75 
the concept of global convergence. In Section 4 we show that no stationary iteration without memory which uses linear information can be globally convergent for the class of all analytic complex functions in Section 5 we extend this result to all stationary iterations with or without memory using linear information In Section 6 we pose a conjecture that for the class of all real polynomials with real simple zeros, there does not exist a globally convergent stationary iteration using linear information.

\section{Stationary Iterations Without Memory}

We recall the definition of information and stationary iteration. (See Traub and Wozniakowski |S|.) For the reader's convenience. in Sections 2 to 4 we deal only with une-point iterations without memory. The extension to the general case is given in Section 5

Consider the solution of a nonlinear scalar equation

$$
f(x)=0
$$

for $f \in F$, where $\mathcal{F}$ is a subset of a space $H$ of functions $f: D, \subset C \rightarrow C$. To solve (2.1) iteratively. we need to know something about $f$. Let $L_{1}: D_{L_{1}} \subset H \times C \rightarrow C$ be a functional which is linear with respect to the first argument; i.e.

$$
L_{1}\left(c_{1} f_{1}+c_{2} f_{2}, x\right)=c_{1} L_{1}\left(f_{1}, x\right)+c_{2} L_{1}\left(f_{2}, x\right) \quad \text { whenever } \quad x \in D_{f_{1}} \cap D_{l_{2}}, \quad i=1,2, \ldots, n \text {. }
$$

Consider the linear information operator $A,+D, C H \times C \rightarrow C^{n}$. defined as

$$
\mathcal{N}(f, x)= \begin{cases}{\left[L_{1}(f, x), L_{2}(f, x), \ldots, L_{n}(f, x)\right]} & \text { for } x \in D_{f} . \\ \text { undefined } & \text { otherwise. }\end{cases}
$$

for every $f \in H$.

Let $x_{0}$ be an approximation of a solution of (2.1). Let $\Phi: D_{\star} \subset C^{n+1} \rightarrow C$ be a functional. We construct the sequence of approximations $x$, by the formula

$$
x_{1+1}=\varphi\left(x_{1} ; \mathcal{N}\left(f, x_{1}\right)\right) \text {. }
$$

The functional $\varphi$ is called a one-point stationary iterative operator without memory using a linear information operator. 4 . For brevity $\Phi$ is called an iteration. Let $\Phi(1)$ ) be the class of all such iterations.

Note that most iterations use values of $f$ and its derivatives. A linear information operator is a generalization of this. For example, the information operator 1 used by Newton iteration,

$$
x_{i+1}=x_{1}-\frac{f\left(x_{1}\right)}{f^{\prime}\left(x_{i}\right)}
$$

is $\mathscr{V}(f, x)=\left[f(x), f^{\prime}(x)\right]$. This operator is linear and $\varphi \in \Phi(\mathscr{N})$.

\section{What Do We Mean bv Global Convergence?}

Let $F$ be the class of all functions $f, f: D, C C \rightarrow C$ analytic in $D$, and having only simple zeros. Let $S(\Omega$ be the set of all zeros of $f$.

Consider any iteration $\varphi \in \Phi$. where $\Phi$ is the class of all stationary iterations without memory which use a linear information operator . per iterative step; i.e., $\Phi=U$. $\Phi(\mathcal{N}$ ).

Which properties should $\varphi$ have to be called a globally convergent iteration? To motivate our definition, consider first the problem $f(x)=0$ where $f$ is defined in $D_{l}=$ $\{x:|x-\alpha|<R(f)\}$ and $\alpha$ is its unique simple zero. Suppose we apply $q$ to this problem Let $r(f, \varphi)$ be the maximal number such that for any starting point $x_{0}$ satisfying $\left|x_{0}-\alpha\right|<r(f, q)$, the sequence $x_{1+1}=\varphi\left(x_{i} . v\left(f, x_{1}\right)\right)$ is convergent to $\alpha$. Then the ball $\{x:|x-a|<r(f, \varphi)\}$ is called the ball of convergence of $\Phi$ for $f$. Of course, $(f, \varphi)$ depends on $R(f)$ and. in particular. $r(f . q) \leq R(f)$. Suppose there exists a positive constant $c=$ $c(\phi)$ such that $r(f, q) \geq c R(f)$ for any $f$ Then $q$ enjoys a type of global convergence, since 
the ball of convergence has radius proportional to the radius $R(f)$ of the domain $D$. However. for problems with $R(f)=+\infty$. we get $n f . q)=+\infty$. which means that any choice of a starting point $x_{0}$ yields convergence. This seems to be too strong. For $R(f)=$ $+\infty$ we would like to have $r(f, q)$ large but not necessarily equal to infinity This is the motivation of the following: Let $K . L$ be two given constan such that $K \geq 0$ and $0<L$ $\leq+\infty$. Define $R_{f}=\min (R(f) . K|a|+L\}$. Now the existence of a positive constant $c=$ $c \Phi)$ such that $r(f, \Phi) \geq c R$, implies a type of global convergence of $q$. This discussion shows that we should compare $r(f, \varphi)$ with $R$,

If $f$ has more than one simple zero, we proceed as follows. Let dist $\left(a, \partial D_{r}\right)$ be the distance of $\alpha, \alpha \in S(f)$, to the boundary $\partial D_{f}$ of the domain $D_{f}$. Define

$$
R_{f}(\alpha)=\min \left(\operatorname{dist}\left(\alpha, \partial D_{l}\right) \cdot K|\alpha|+L\right), \quad(K \geq 0,0<L \leq+\infty) .
$$

Note that if $S(N)=\{\alpha\}$. then $R_{f}(\alpha)=R_{f}$. Let

$$
B(b, f)=\bigcup_{x \rightarrow 1, j}\left\{x:|x-a|<b \cdot R_{f}(\alpha)\right\}
$$

where $b \geq 0$. For any iteration $\varphi . \varphi \in \Phi\left(.1^{\circ}\right)$, we define a number $c(\varphi)$ such that

(i) for any $f \in \mathcal{F}$ and for any staring point $x_{0} \in B(C(\varphi) . f)$, the sequence $\left\{x_{1}\right\}, x_{1+1}=$ $\varphi\left(x_{1}, f^{*}\left(f, x_{1}\right)\right)$, is well defined and $\lim _{1 \rightarrow \infty} x_{1} \in S(f)$; and

(ii) for any $\epsilon>0$, there exist a problem $f \in$ and a starting point $x_{1} \in B(c(\varphi)+\epsilon$. $f)$ such that either the sequence $\left(x_{1}\right)$ is not well defined or $\lim _{t \rightarrow} x, E S(f)$

Note that for any iteration, $c(\varphi) \in[0.1]$. The set $B(c(\varphi), f)$ is a convergence domain of $\varphi$ for the function $f$, since taking any starting point $x_{0} \in B(c(\varphi)$. $f)$ we get convergence of $\left\{x_{1}\right)$ Note, however, that we do not specify which element from $S(f)$ is the limit of $\left\{x_{i}\right\}$.

Definition 3.1. We shall say that an tteration $q . q \in \Phi$. is globally convergent for the class $F$ iff $C(\Phi)>0$.

Definition 3.1 imposes only a weak condition on 9 However. we shall shou that for any iteration from $\Phi . c(\tau)=0$. This means that even in the sense of Detinition 3.1 there extsi no globally convergent stationary iteration using linear information for the class $\bar{p}$.

\section{Main Result}

THEOREM 4.1. No iteration $\Phi$ from $\Phi$ is globally convergent for the class.

Proof. Suppose there exist. $1: D, \rightarrow C^{n}$ and $\varphi \in \Phi(1)$ with $\left.c=c \varphi\right)>0$ Let

$$
a= \begin{cases}1 & \text { if } c K \geq 1 . \\ \frac{c L}{2(1-c K)} & \text { if } c K<1 .\end{cases}
$$

Define $f(x)=x-a$. Then $f \in \tilde{F}, S(f)=\{a\}$. and $R_{f}(a)=K|a|+L$. Let $x_{t \prime}=0$. Since $x_{0} \in B(c, f)=\left\{x:|x-a|<c R_{r}(a)\right\}$. the sequence $\left.\left\{x_{i}\right\}, x_{i+1}=q\left(x_{i}, 1 / f, x_{i}\right)\right)$. tends (1) $a$. $a \neq 0$. Thus there exists a unique integer $k . k \geq 1$, such that

$$
\left|x_{0}\right| .\left|x_{1}\right| \ldots\left|x_{1}\right|<\frac{a}{2} \quad \text { and } \quad\left|x_{1}\right| \geq \frac{a}{2}
$$

Consider a polynomial $s$ of the form

$$
n(x)=\sum_{i=0}^{n+k} a_{i} x^{1+1}
$$

which satisfies

$$
1\left(f-n, x_{\jmath}\right)=1\left(f, x_{1}\right) \text { for } \jmath=0.1 . \ldots k-1
$$


This is equivalent to the following homogeneous system of $n \cdot k$ linear equations:

$$
\sum_{i=0}^{n} a_{1} L_{d}\left(x^{1+1}, x\right)=0 \text { for } s=1.2 \ldots n \text { and } j=0.1 \ldots k-1
$$

Since we have more unknowns than equations, it is obvious that there exists a nonzero polynomial $w$ of the form (4.2). Then there exist positive integers $r, 1 \leq r \leq n k+1$, and $p$. $I \leq p \leq r$, and nonzero $z_{p+1} \ldots . .2$, such that

$$
m(x)=x^{p}(x-2 p+1) \cdot \cdots \cdot(x-2,)
$$

For $\epsilon>0$. define

$$
f(x)= \begin{cases}f(x)+\frac{1}{\epsilon} n(x) & \text { if }|x|<\frac{a}{2} \\ \text { undefined } & \text { if }|x| \geq \frac{a}{2}\end{cases}
$$

From the general theory of algebraic functions (see. e.g. Wilkinson [7]), it is known that for sufficiently small $\epsilon$ there exist $p$ simple zeros $\lambda_{1}(\epsilon) \ldots, \lambda_{f}(\epsilon)$ of $f$, such that

$$
\lambda_{1}(\epsilon)=\left|\frac{p^{\prime} f(0)}{w^{\prime p+}(0)}\right|^{1, \prime} \epsilon_{1}+O\left(\epsilon_{1}^{2}\right)
$$

where $\epsilon_{\text {, is }}$ the $i$ th complex $r(x)$ of the equation $r^{\prime \prime}=\epsilon$. Note that lim, $\lambda_{0}(\epsilon)=0 . \forall$ t Therefore, for sufficiently small $\epsilon$, we get $f, \in, \lambda_{1}(\epsilon), \lambda_{3}(\epsilon), \ldots, \lambda_{p}(\epsilon) \in S\left(f_{0}\right)$ and $\lim _{1 \rightarrow 0} R_{1}\left(\lambda_{1}(\epsilon)\right)=\min \{a / 2$. L $\}$. Then the staring point $y_{0}=x_{0}=0$ belongs to $B\left(c, f_{1}\right)$. This means that the sequence $\left.\left.\left\{v_{1}\right\}, v_{1}=q\left(v_{1}, 1\right\} f_{1}, v_{1}\right)\right)$. is well defined Ohserve that $+\left(f_{1}, x_{j}\right)=t\left(f_{0 .} y_{j}\right)$ for $j=0,1, \ldots, k-1$ and $y_{0}=x_{0}$. Therefore $y_{i+1}=x_{1+1}=$ $\varphi\left(x_{1}, n f\left(x_{1}\right)\right)$ for $i=0.1 \ldots, k-1$. From (4.1) we know that $\left|y_{k}\right|=\left|x_{k}\right| \geq a / 2$. which means that $y_{4}$ does not belong to the domain $D_{f_{1}}$. Thus. $l\left(f_{1}, y_{k}\right)$ is not well defined (see (2.2)). which contradicts $(F)>0$.

Theorem 4.1 says that there exists no globally convergent iteration without memory using a linear information operator for the class .7 of analytic complex functions having only simple zeros. This negative result also holds for real problems. Let $F_{1}$ be the class of real functions $f, f: D, \subset R \rightarrow R$, analytic in $D$, and having a unique simple zero. Consider real information operators, i.e... $(f, x) \in R^{n}, \forall f \in S, \forall x \in D_{\text {f. }}$

THEOREM 4.2. For any real linear information operalor \& there exisis no llezation $\$$ from $\Phi .1$ ) which is globally convergent for the class.

Proof. Suppose that for a real linear information operator $r .,: D, \rightarrow R^{n}$, and for $\Phi \in \Phi(1), c=c(\Phi)>0$. Let $\lambda(x)=x-\sigma$ be defined as in the proof of Theorem 4.1. Since $x_{0}=0 \in B(c, f)$, the sequence $\left\{x_{1}\right\}, x_{1 \rightarrow 1}=\Phi\left(x_{i}, f\left(f, x_{1}\right)\right)$ tends to $a \neq 0$. Therefore

$$
x_{1}=\phi(0.4(f .0)) \neq 0 \text {. }
$$

Consider now a real polynomial w.

$$
m(x)=\sum_{i=n}^{n} a_{i} x^{2+1}
$$

satisfying

$$
(f-n, 0)=1(f, 0)
$$

The equation (48) is equivalent to the following homogeneous system of $n$ real linear equations:

$$
\sum_{i=0}^{n} a_{i} L_{d}\left(x^{2+1}, x_{n}\right)=0 \quad \text { for } \quad s=1,2 \ldots n \text {. }
$$


It is obvious that there exists a nonzero real polynomial $w$ of the form $(4,7)$ and satisfying (4.8). Let $m(x)=x^{\prime \prime}\left(x-z_{p \cdot 1}\right) \cdot \ldots \cdot\left(x-z_{1}\right)$ where $1 \leq r \leq 2 n+1.1 \leq p \leq r$. and $z \neq u$ for $t=p+1, \ldots, r$ Owing to $(47), p$ is odd

Define

$$
\Gamma= \begin{cases}\min \left\{\frac{\left|x_{1}\right|}{2} \cdot \frac{\left|z_{p+1}\right|}{2} \ldots, \frac{|z|}{2}\right\} & \text { if } p<r \\ \frac{\left|x_{1}\right|}{2} & \text { otherwise. }\end{cases}
$$

and

$$
f(x)=\left\{\begin{array}{lll}
f(x)+\frac{1}{\epsilon} m(x) & \text { if }|x|<\Gamma \\
\text { undefined } & \text { if }|x| \geq \Gamma
\end{array}\right.
$$

for $\epsilon>0$. For sufficiently small $\epsilon$. the solutions of $f(x)=0$ in the complex plane are given by (4.5). Since $p$ is odd, only one of $\lambda_{1}(\epsilon)$ in (4.5) is real. Thus $f$. has a unique simple real zero, say $\lambda(\epsilon)$. Since $\lambda(\epsilon)$ tends to zero with $\epsilon, \lim , \rightarrow 0 R_{f}(\lambda(\epsilon))=\min (\Gamma, L)$, and $x_{0}=0$ $\in B\left(c, f_{0}\right)$ for sufficiently small $\epsilon$. Therefore the sequence $\left\{x_{1}\right\}, x_{1+1}=q\left(x_{1}, r\left(f_{0}, x_{1}\right)\right)$ is well defined. Observe that

$$
x_{1}=\varphi\left(0 ;+\left(f_{0}, 0\right)\right)=\varphi(0 ;-t(f, 0)) .
$$

Owing to (4.6) $x_{1} \in D_{l}$, which means that $x_{2}$ is not well defined. This contradiction ends the proof.

\section{The General Case}

In Section 4 we showed that there exists no globally convergent one-point stationary iteration without memory which uses linear information. In this section we prove the same result for multipoint iterations with or without memory

Let $L_{1} . L_{2}, \ldots L_{n}$ be functionals defined as in Section 2. Then a multipoint linear information operator $1: D, \subset H \times C \rightarrow C^{n}$. is defined as

$$
f(f, x)=\left[L_{1}\left(f, z_{1}\right), L_{i}\left(f, z_{i}\right), \ldots \ln \left(f, z_{n}\right)\right] . \quad \forall f \in H, \quad \forall x \in D_{1}
$$

where

$$
z_{1}=x \quad \text { and } \quad z_{k}=\xi_{k+1}\left(z_{1}, L_{1}\left(f, z_{1}\right), L_{i}\left(f, z_{2}\right) . \quad . L_{k}\left(f, z_{k}\right)\right)
$$

$$
k=1,2, \ldots n-1
$$

for cerrain functions $\xi_{2} . \xi_{3} \ldots . \xi_{n}$. Note that if $\xi_{k}=x . k=2, \ldots, n$, then .1 is a one-point linear information operator as defined in Section 2

For given integer $m$. let $x_{1} . x_{1} \ldots \ldots x, m$ he distinct approximations of a solution of $f(x)=0, f \in F$. Suppose we construct a sequence of approximations by the formula

$$
x_{1+1}=\Phi\left(x_{1}, x_{1-1} \ldots \ldots, x_{m}, t\left(f, x_{1}\right), 1\left(f, x_{1}\right), \ldots,\left(f, x_{1}\right)\right) \text {. }
$$

where $q: D_{*} \subset C^{(m+1) i n+11} \rightarrow C$ is a functional. Then $\Phi$ is called a mullipoint stationar: Iteration operator with memory if $m \geq 1$ and wuhout memorv if $m=0$ using a linear information operator. 1 . For brevily $q$ is also called an tteration. Let $\Phi_{m}(1$ ) be the class of all such iterations.

For particular. 4 and $m$ we get commonly used tlerations. For instance. $m=1, f(f, x)$ $=[\Omega(x)]$. and

$$
\varphi(x, y:,(f, x), .(f, y))=x-\frac{x-y}{\Omega(x)-\jmath(y)} f(x)
$$

is the secant iteration. An example of two-point stationary iteration without memory is provided by Steffensen iteration [2]. which is defined as follows

$$
\left.f(f, x)=\left[f\left(z_{1}\right), f\left(z_{1}\right)\right] \text { where } z_{1}=x \text { and } z_{2}=z_{1}-\lambda_{i_{1}}\right)
$$


and

$$
q(x:+(f, x))=z_{1}-\frac{z_{1}-\ddot{z}_{2}}{f\left(z_{1}\right)-\rho\left(z_{2}\right)} f\left(z_{1}\right) .
$$

An example of a multipoint method with memory can be found in Traub 13 . Pp. 185-186].

We extend the definition of global convergence as follows. Let. 1 be a one- or multipoint linear information operator and let $m$ be an integer. Recall that $B(b, f)$ is defined in Section 3. For any iteration $q . q \in \Phi_{m}(\cdot)$. define $c=c(\Phi)$ as a number such that

(i) for any $f \in F$ and any choice of distunct staring points $x_{0}, x_{-1} \ldots, x_{-m}$ from $B(c, f)$, the sequence $\left(x_{1}\right\}, x_{i+1}=\Phi\left(x_{1}, x_{i-1} \ldots, x_{1-m i} \mathcal{N}\left(f, x_{1}\right), \ldots\left(f, x_{1-1}\right), \ldots\right.$ .$v\left(f, x_{1-m}\right)$ ) is well defined and $\lim _{-x} x_{1} \in S(f)$;

(ii) for any $\epsilon>0$ there exist $f \in$ and distinct points $x_{0,} x_{-1}, \ldots x_{-m} \in B(c+\epsilon . f)$ such that either the sequence $\left(x_{1}\right)$ is not well defined or $\lim _{1-\infty} x_{1} \notin S(f)$.

Definition 5.1. We shall say that an iteration $\varphi$ is globally convergent for the class $F$ if $c(\varphi)>0$.

Now let $\Phi$ be the class of all stationan iterations with or without memory which use a linear information operator $v$; i.e., $\Phi=U, U_{m} \Phi_{m}(r)$.

\section{THEOREM S.I. No iteration $\varphi$ from $\Phi$ is globally convergeni for the class $\boldsymbol{F}$.}

Phoof. Apply the proof of Theorem $\$ 1$ with starting points

$$
\left.x_{-1}=\frac{j}{4(m+1)} \min : a, L\right\}, \quad j=0,1 \ldots, m .
$$

and with $n \cdot k$ in $(4.2)$ replaced by $n(m+k)$.

Theorem 5.1 says that knowing only the value of a finite number of linear functionals on $f$ it is impossible to find a globally convergent stationary iteration for the class 7 . Thercfore, if we want 11 wilve $f(1)=0$ hy is statiunary iteration. we huve to assume that the starting points are sufficiently close to a solution. By contrast it is known that for some nonlinear information operators there exist globally convergent stationary iterations. An example is provided by Laguerre iteration for the class of all real polynomials with simple zeros. Also, for linear information uperators. there exist globally convergent nonstarionury. iterations for the class $\tilde{F}$. An example may be adopted from Traub and Wozniakowski [4], where global convergence of the sequence of interpolatory iterations $I_{k}$ is proved. For this case, the $k$ th iteration requires the knowledge of $k$ linear functionals of $f$. This with be reported in Wasilkowski $[6]$

\section{Final Comments}

In Section 5 we showed that no stationary iteration using linear information is globally convergent for the class of analytic problems having simple zeros. The existence of globally convergent iterations depends on the class, of functions whose zeros we want io approximate. Fur sume simple classes there exist well-knuwn globally convergent stationary iterations which use linear information For example. if is the class of real functions $f: R \rightarrow R$ whose first derivative is monolunic. then Newton iteration is globally convergent.

For many inleresting clisses the exwence of global convergent iterations is unknown. Even for the class II of all real polynomials with simple real zeros, this problem is open. All knuwn globally convergent iterations for the class $\Pi$ are either nunstationary or use nonlinear information. For example. Vewton tteration with a suitably chosen staning point. Bernuallis methed. and Litguerre neration are globally convergent for II. (See. e.g.. Ralsion and Rahinowizz [1].) Fither implicitly or explicitly these iterations use the degree $k$ of the polynomial whose zero is desired 
Note that the degree $k$ is nonlinear information. Indeed. suppose there exists a function $g$ (in general nonlinear) such that $k=g(x: 1(f, r)$ ) where 1 is a multupoint linear information operator. For any $x_{u}$ there exists a polynomial $w$ of degree greater than one such that $f\left(w, x_{0}\right)=0$. Taking $f_{1}(x)=x+(\operatorname{l} f \epsilon) w(x)$, we gel,$\left(f_{0}, x_{0}\right)=f\left(x, x_{0}\right)$ and $f, \in \Pi$ for sufficiently small $\epsilon$. Therefore $g\left(x_{m} . \downarrow\left(x, x_{0}\right)\right)=g\left(x_{11} . \downarrow\left(f_{1}, x_{11}\right)\right)$. but the degrees of the polynomials $x$ and $f_{1}(x)$ are different. This contradicts the assumption $k=$ $g(x ;+\forall(f, x))$, with 1 linear.

We believe that any globally convergent iteration for $\Pi$ has to be nonstationary or use nonlinear information. Therefore we propose the following conjecture.

Conjecture 6.1. There exists no globally convergent stationary iteration using linear information for the class of all real polynomials with simple real zeros.

ACKNOWLEDGMENTS. I thank J F. Traub and $H$. Wozniakowski for their help Without it this paper could not have been completed.

\section{REFERENCES}

1. Ralstor. A. And Rabinowitz. P a Firs! Course in Nimerical Analysis. 2nd ed. McGraw Hill. New York 1978

2. STEFrENSEN, J.F. Remarks on iteration. Skand. Aktuar. Tidsh 10 (1933), 64-72.

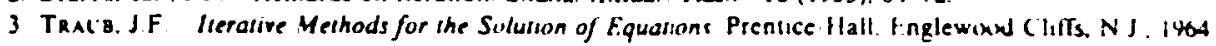

4. TraUd. J.F. AND WOZniakowski, H. Optimal radius of convergence of interpolatury iterauons for operator equations Dept Comptr Sci Rep. Carnegie-Mellon U 1976 To appear in Aequariones Muthemilicoe

S. TRAUB. J.F., AND WOZNIAKOWSK., H. General theory of optimal error algonthms and analytic complexily. Pan B: Iterative information mudel Depi Compir Soi Rep Carnegie-Mellon U. 1978 To appear in A General Theory of Opumal Algorihms. Academic Press. New York. 1980

6 Wasilxowiski, GW The sirength of nonstationary iteration Depi Compit Sa. Rep. Camegie.Mellon U. 1979

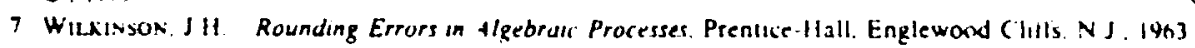

RECEIVED IANLARY 1979. REVISED APRIL 1979: ACCEPTED MAY 1979 